\title{
Surgical management of intestinal failure
}

\author{
G. L. Carlson \\ Intestinal Failure Unit, University of Manchester, Hope Hospital, Eccles Old Road, Salford M6 8HD, UK
}

\begin{abstract}
Surgery plays a key role in the management of both acute and, less frequently, chronic intestinal failure. Acute intestinal failure frequently requires surgical treatment when it arises as a consequence of intestinal fistulation or obstruction. In specialised clinical practice approximately $50 \%$ of acute intestinal failure is associated with intestinal fistulas and in approximately $50 \%$ of patients, this condition arises as part of the natural history or complicating treatment for Crohn's disease. A considerable proportion of such patients have abdominal infection and present complex nutritional and metabolic problems. The most important aspect of the surgical management of patients with acute intestinal failure associated with intra-abdominal infection is management of sepsis, since recovery is unlikely in the presence of active infection. Moreover, effective nutritional support and restoration of body composition is not possible if sepsis remains unresolved. Surgical strategies to deal with intra-abdominal infection may involve percutaneous drainage, laparotomy and resection of fistulating segments of intestine and, when infection is persistent and contamination extensive, laparostomy (a technique in which the abdomen is left open and allowed to heal by secondary intention). Surgical treatment should not only be timely and effective, but also aimed at preventing secondary damage to the small intestine, in order to minimise the risk of short bowel syndrome. In some cases a proximal defunctioning stoma may be required, with prolonged nutritional support, using either home total parenteral nutrition or feeding via the defunctioned distal gut (fistuloclysis), pending restoration of intestinal continuity. The role of surgical treatment for patients with short bowel syndrome is less clear. While surgery is frequently required for the management of complications of short bowel syndrome (including gallstones and possibly peptic ulcer disease), the role of intestinal lengthening and tapering procedures (to increase functional intestinal length), and artificial valves, reversed segments and colonic interposition (to reduce intestinal transit) remains controversial. For some patients with short bowel syndrome and, in particular, those with combined intestinal and hepatic failure, intestinal transplantation may become the treatment of choice as long-term results continue to improve.
\end{abstract}

\section{Intestinal failure: Intra-abdominal infection: Surgical management:} Sepsis management: Nutritional support

Intestinal failure is defined as a reduction in functioning gut mass below the minimum amount necessary for adequate digestion and absorption of nutrients (Fleming \& Remington, 1981), although later authors have added failure of the intestinal tract to maintain hydration and electrolyte balance in the absence of supplements (Nightingale, 2001). Failure of the intestinal tract to maintain life (or at least health) in the absence of artificial nutritional support may arise from a variety of disease processes and insults, but typically occurs in the form of two separate clinical syndromes. Acute intestinal failure is common, frequently reversible and often related, or at least attributable, to conditions that are managed by surgeons. In contrast, chronic intestinal failure is less common, typically irreversible with currently available treatments (thus necessitating lifelong artificial nutritional support) and when it does arise, frequently does so as a consequence of the complications of surgical treatment. The purpose of the present review is to examine the role that surgical treatment plays in the management of both acute and chronic intestinal failure, with an emphasis on the treatment of adult patients.

\section{Acute intestinal failure}

Acute intestinal failure is exceedingly common and is routinely managed by virtually all general surgeons. The vast 
majority of cases are transient and follow a short-lived clinical course with spontaneous resolution. They are either managed without any form of nutritional support or with nutritional support for $<14 \mathrm{~d}$. Acute intestinal failure under these circumstances may develop for a variety of different reasons (see Table 1).

In contrast, the annual incidence of complicated intestinal failure requiring referral to specialised intestinal failure units is of the order of 5.5 new patients per million of the population in the UK (Scott et al. 1991). This level of incidence may, however, be an underestimate of the true incidence of complex acute intestinal failure, since many cases will have been managed in non-specialist centres. The advent of clinical governance and the decision by the Department of Health to recognise acute intestinal failure as a distinct clinical entity and to provide centralised funding for the provision of health care for such patients has tended to increase the number of patients referred for the management of acute intestinal failure. Appropriate referral criteria have been established to indicate which patients should be offered specialised management (Table 2).

Although many cases of acute intestinal failure on the ordinary surgical ward occur as a consequence of simple intestinal obstruction, post-operative fistulation etc., the spectrum of disease that presents for specialist management is markedly different, primarily because uncomplicated cases

Table 1. Causes of acute intestinal failure (adapted from Pettigrew \& Hill, 1984)

When the gastrointestinal tract is 'blocked'

Mechanical obstruction

Paralytic ileus

Intestinal pseudo-obstruction

When the intestinal tract is too short

Massive resection

Internal or external fistulas

When the intestinal tract is inflamed

Inflammatory bowel disease

Severe infective enteritis

Radiotherapy

Chemotherapy

When the gastrointestinal tract fails to function adequately for other reasons

Intra-abdominal sepsis

Multiple organ failure

Acute pancreatitis

Table 2. Guidelines for referring patients with acute intestinal failure to a specialist unit (Carlson, 2001)

Persistent intestinal failure for $>6$ weeks complicated by venousaccess problems

Multiple fistulas within a totally-dehisced abdominal wound

Total or near total ( $<0.30 \mathrm{~m}$ remaining) enterectomy

Recurrent venous-access problems due to sepsis or thrombosis

Persistent severe abdominal sepsis

Persistent nutritional or metabolic problems associated with a high-output stoma or fistula

A patient with an intestinal fistula beyond the expertise of the referring hospital of acute intestinal failure have usually resolved spontaneously. In contrast, the majority of cases ( $>60 \%)$ referred for specialist treatment will require surgical intervention (Scott et al. 1991).

The single most common diagnosis associated with the development of acute intestinal failure requiring surgical treatment is Crohn's disease (Fig. 1), because of the development of intestinal fistulas, either as a consequence of the disease itself or as a complication of surgical treatment (Fig. 2).

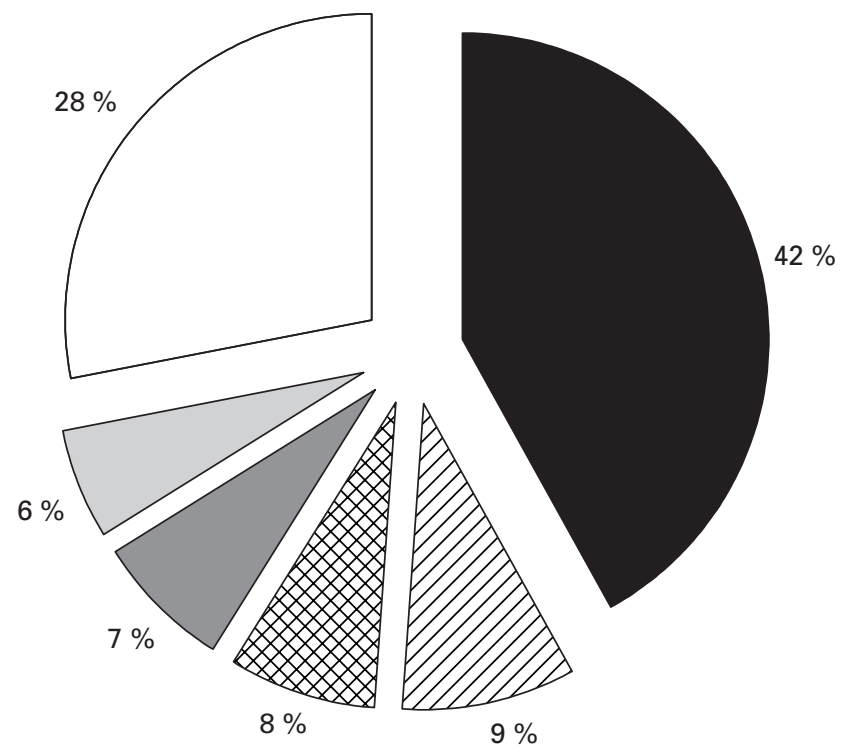

Fig. 1. Aetiology of acute intestinal failure in a specialised unit. ( $\square$ ),

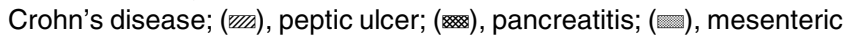
vascular disease; (品), malignancy; $\square$ ), other. (Adapted from Scott et al.1991.)

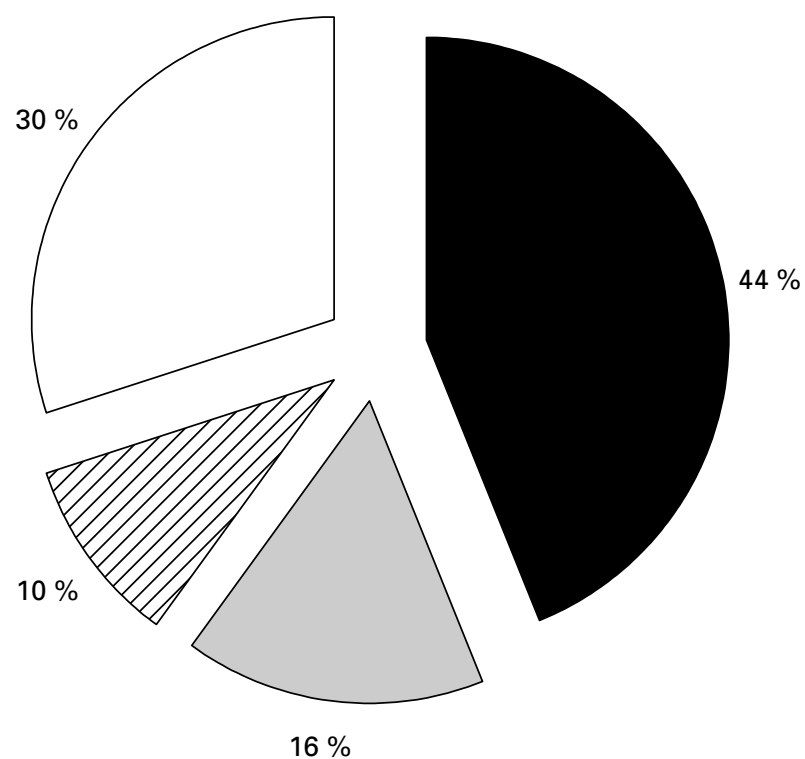

Fig. 2. Clinical problems associated with the development of acute

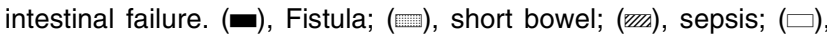
other. (Adapted from Scott et al. 1991.) 


\section{Principles of surgical management}

\section{Treatment of abdominal sepsis}

Abdominal sepsis commonly accompanies acute intestinal failure, particularly when associated with the development of intestinal fistulas (Carlson, 2001). Rapid and effective diagnosis and management of sepsis is probably the most important single measure in the surgical care of the patient with acute intestinal failure, for a number of reasons. Inadequately-treated abdominal sepsis leads to progressive impairment of organ function and ultimately death from multiple organ failure syndrome. Spontaneous healing of fistulas is unlikely to occur in the presence of an associated abscess cavity and active infection (Carlson \& Irving, 2000). This condition results in sustained increased energy requirements (Carlson, 1998) and impaired fuel substrate utilisation and insulin resistance (Carlson \& Little, 1992, 1994), and leads to progressive inanition and loss of lean body mass. The result is a state of cachexia that may resemble advanced malignant disease, despite aggressive nutritional support (Carlson \& Irving, 1997).

It has been recognised for many years that sepsis is the most common cause of death in patients with complicated acute intestinal failure (Soeters et al. 1979). Attempts to diagnose, localise and treat abdominal sepsis should take first priority in the clinical assessment of all patients with acute intestinal failure. Although the key diagnostic criteria for the systemic inflammatory response syndrome and sepsis are well established (Bone et al. 1992), it is important to note that in many patients with long-standing walled-off abdominal infection, the cardinal features of pyrexia, leucocytosis tachycardia and tachypnoea are frequently absent. Cachexia, hypoalbuminaemia, jaundice and hyponatraemia are more characteristic of patients who may have had low-grade intraabdominal infection for weeks or even months, although major surgical intervention in these apparently-stable patients may lead to the rapid and catastrophic development of sepsis syndrome, multiple organ failure and death. Abdominal infection should be investigated with contrast-enhanced computed tomography, which in experienced hands has a diagnostic accuracy of $\geq 97 \%$ (Gerzof \& Oates, 1988). Alternative techniques, including ultrasound, magnetic resonance imaging and labelled leucocyte scanning may be of value in some patients, but have not been shown to be superior to computed tomography scan. Identification of a focus of sepsis in the peritoneal cavity of a patient with acute intestinal failure should lead to prompt drainage. Antibiotic therapy may be of value, but will not generally allow complete resolution of abdominal sepsis if there is an established abscess cavity. Where possible, abscesses should be drained under radiological control by percutaneous drainage. This treatment may avoid the morbidity and mortality of the 'second hit' associated with surgical trauma in a patient with sepsis (see earlier). Radiological drainage may require the insertion of several large-bore drains (10 Fr, including irrigation catheters), which can be removed gradually once abscess cavities have been shown by contrast radiology to have collapsed. In cases in which there is complete intestinal discontinuity radiological drainage alone will not gain control of infection, and resection of the relevant intestinal segment, or at least proximal diversion, will be required. Surgical treatment will be required where sepsis is associated with marked intestinal discontinuity, and where there are multiple loculi of infected fluid that cannot be drained by percutaneous means (e.g. when there are multiple abscesses between bowel loops). Under these circumstances, sometimes referred to as tertiary peritonitis, a variety of surgical approaches have been adopted, including radical peritoneal debridement (Polk \& Fry, 1980) and continuous lavage of the peritoneal cavity with antibiotic solutions (Jennings et al. 1982). Neither of these techniques has been shown to be effective, nor has planned relaparotomy in patients with severe abdominal sepsis (as opposed to laparotomy on demand; Hau et al. 1995).

There are several surgical strategies appropriate to the management of abdominal sepsis associated with intestinal fistulas. When sepsis occurs in the presence of a fistulating segment of intestine, the segment should be resected if possible and the ends exteriorised as stomas. If resecting the segment of intestine would jeopardise other apparently healthy loops of intestine, drainage and proximal diversion of the gastrointestinal tract may be appropriate. If there is extensive peritoneal contamination with multiple interloop abscesses, and the fistulating segment of bowel is fixed within dense inflammatory tissue, it may be safer to leave the entire abdomen open and drain the fistulating segments (laparostomy; Mughal et al. 1986), allowing the abdomen to heal by secondary intention. The ability to manage cases of complex acute intestinal failure associated with abdominal sepsis by creating proximal intestinal stomas or large laparostomy defects and multiple fistulas is highly dependent on extensive nursing expertise and support and, until recently, safe and effective total parenteral nutrition (TPN), to allow patients to recover pending definitive intestinal reconstructive surgery (see p. 714).

\section{Skin care}

Intestinal fluid may be acidic or alkaline, depending on the nature of the fistula, and may also contain corrosive enzymes. Inadequate protection of the skin around enterocutaneous fistulas may lead to painful and progressive digestion, with secondary infection. There are also few issues more demoralising for patients with intestinal fistulas than an inability to control fistula output. The specialist nurse and/or enterostomal therapist have a key role to play in the management of the skin around fistulas, and a variety of stoma appliances, suction catheters and adhesive paste dressings are available to aid fistula management (Hughes et al. 2001). In cases in which there is a fistula deep within an irregularly-shaped wound, it may be necessary to bring out a proximal loop jejunostomy, the effluent from which can at least be diverted into a properly-fitting stoma appliance.

\section{Provision of safe and effective nutritional support}

The aim of nutritional support for the patient with acute intestinal failure is to maintain adequate nutritional status until the cause of acute intestinal failure has either resolved spontaneously (e.g. a fistula has healed) or has been surgically corrected. In addition, many patients with acute intestinal failure will be nutritionally depleted, and nutritional support in these patients is required for restoration of 
lean body mass and to optimise recovery from surgical treatment. It has been repeatedly shown that it is not possible to provide effective nutritional support in the presence of active infection (Shaw \& Koea, 1993; Streat et al. 1987), hence the initial emphasis on the management of sepsis rather than nutritional support. In general, nutritional support in patients with acute intestinal failure should be considered whenever starvation for $>5 \mathrm{~d}$ is expected, has already occurred as a result of impaired food intake or gastrointestinal disease, if complications (e.g. sepsis) have increased energy requirements beyond those that can be provided by a normal diet, or there was already nutritional depletion before the development of acute intestinal failure (Chandran \& Sim, 1991).

In practice (and by definition) virtually all patients with acute intestinal failure will require nutritional support. In simple cases associated with post-operative intestinal dysfunction this support will only be necessary until digestive function has returned, whereas in patients with high-output fistulas in the proximal gastrointestinal tract nutritional support will be required until fistula closure has occurred or corrective surgery successfully completed. In patients who have already had extensive surgery, and especially in those with a fistula and sepsis in whom the abdomen has been left open, it may take many months before further attempts at correction of the fistula can be planned, because of the time taken for the peritoneal cavity to become re-established (Scripcariu et al. 1994). In general, such patients are maintained on TPN until definitive surgery can be undertaken, although in selected patients with mucocutaneous continuity feeding through the fistula may be feasible, safe, effective and potentially less expensive than parenteral nutrition. Traditionally, most patients with acute intestinal failure are fed parenterally and, indeed, the concept of an intestinal failure unit has developed around a cohort of specialist nursing staff who could ensure that TPN could be delivered with minimal morbidity and mortality. If TPN is likely to be required for $<14 \mathrm{~d}$ only, it may be possible to provide energy and $\mathrm{N}$ requirements using a catheter inserted in a peripheral vein. The chief attraction of the peripheral route for TPN is that it requires little special expertise and tends to have a lower associated morbidity than intravenous feeding through the central venous route. The duration of intravenous feeding tends, however, to be limited by the development of thrombophlebitis, principally as a result of the low $\mathrm{pH}$ and high osmolality of TPN solutions (May et al. 1996). The development of thrombophlebitis can be minimised by the use of lipid as the principal energy source, since the presence of lipid considerably reduces the osmolality of the TPN solution. In addition, the higher energy density of lipid effectively means that energy requirements can be met with much lower feed volumes, thus reducing the potential exposure of the venous endothelium to feeding solutions. Additional measures that may facilitate peripheral parenteral feeding include the use of dedicated ultrafine-bore catheters made of inert materials (such as polyurethane), which interfere less than traditional catheters with blood flow in the vein (Plusa et al. 1998; Williams et al. 1996), addition of heparin and hydrocortisone to the feeding solutions and the use of nitrate patches to promote venodilatation and improve local blood flow (Tighe et al. 1995). In many cases, and especially in those patients with a high-output ( $>500 \mathrm{ml} / \mathrm{d}$ ) intestinal fistula (Carlson \& Irving, 2000) and associated abdominal sepsis, the large fluid, energy and $\mathrm{N}$ requirements and the prolonged requirements for nutritional support mitigate against the use of the peripheral venous route for nutritional support. In selected patients it may be possible to utilise the intestinal tract for nutritional support, despite the presence of intestinal failure. In cases in which there is mucocutaneous continuity at the site of an intestinal fistula (which indicates that spontaneous closure will not occur), the intestine distal to the fistula can be intubated and used to deliver enteral feed (Levy et al. 1988), a technique termed 'fistuloclysis'. While early studies used re-infusion of the effluent from the proximal limb of the fistula, this method has proved unpopular and nutritional status can be maintained and, where relevant, improved with polymeric feed and without chyme infusion, provided a sufficient $(>0.75 \mathrm{~m})$ length of healthy small intestine is available distal to the site of the fistula.

\section{Definition of intestinal anatomy}

Once sepsis has been eliminated and the patient's nutritional status is being addressed, consideration should be given to assessing in detail any anatomical abnormalities associated with the development of intestinal failure. The most useful investigations involve intubation of the fistula openings (if present) and detailed contrast radiographic examination by an experienced radiologist. If there are several fistulas, a careful assessment of the timing of contrast studies is required in order to avoid confusion, and generally studies of the intestine proximal to fistula openings should be performed first, in order to avoid loops of bowel filled with the material remaining from investigations distal to fistulas. It is vital to have a thorough understanding of the quantity and quality of bowel remaining before undertaking surgical reconstruction, and especially to rule out strictured segments distal to fistula openings that could lead to anastomotic dehiscence after intestinal re-anastomosis. In some cases, depending on the cause of acute intestinal failure, intravenous urography, percutaneous transhepatic cholangiography and retrograde endoscopic cholangiopancreatography may need to be undertaken to effectively delineate the relevant anatomy.

\section{Definitive treatment}

The definitive treatment required for a patient with acute intestinal failure is determined by the underlying cause. As indicated earlier, many episodes of acute intestinal failure are self-limiting in nature and recover spontaneously without the need for anything other than supportive treatment. Spontaneous closure of intestinal fistulas occurs in $\leq 60 \%$ of cases, usually within 6 weeks of supportive therapy (Carlson $\&$ Irving, 2000). During this period oral fluid and diet should be curtailed, although the role of other strategies is less clear. In particular, although analogues of somatostatin, including octreotide, have been said to promote fistula closure, much of the evidence to support their use is anecdotal and more rigorously controlled randomised trials have failed to provide evidence of benefit. It seems likely that octreotide may increase the speed of fistula closure, but less likely that 
Table 3. Factors associated with failure of an intestinal fistula to heal spontaneously

\begin{tabular}{l}
\hline Local factors \\
Obstruction distal to the fistula site \\
Epithelial continuity across the fistula \\
Mucocutaneous continuity \\
Internal fistulation (e.g. vesicocolic) \\
Associated abscess cavities \\
Disease of the bowel at the fistula site (e.g. Crohn's disease, \\
radiation enteritis) \\
Extensive discontinuity of the bowel ends \\
Systemic factors \\
Sepsis \\
Malnutrition \\
Connective tissue diseases
\end{tabular}

octreotide could promote closure of fistulas where local factors (e.g. distal obstruction) dictate fistula patency. Fistulas are particularly likely to close when they are lateral fistulas (i.e. perpendicular to the long axis) in healthy intestine, and unlikely to close when local and systemic factors make anatomy unfavourable or create systemic conditions in which healing is likely to be impaired (see Table 3). Where it is clear from radiological studies or clinical inspection that spontaneous fistula closure is not possible, there is little point in restricting oral intake, except to facilitate skin care and fluid balance. Surgical treatment for patients whose fistulas do not close spontaneously should be deferred until there has been complete recovery from sepsis and adequate nutritional status has been regained. In addition, local conditions within the peritoneal cavity need to be conducive to complex and technically-demanding reconstructive surgery. Achieving these conditions may require a period of at least 3 months where the abdomen has been closed, and as long as 6 months when the abdomen has been left open and healed by secondary intention (Scripcariu et al. 1994). This outcome frequently requires patients with complex intestinal fistulation to be discharged on home parenteral nutrition pending definitive surgical treatment. The eventual appearance of prolapse in fistulating intestinal loops that had been previously fixed within granulation tissue is an extremely valuable guide to the re-establishment of the peritoneal cavity and suggests that conditions within the abdomen will be conducive to reconstruction (Carlson, 2001). Finally, a considerable amount of psychological support is required to regain the confidence of many patients in their medical and nursing attendants and to prepare them for a further complex operation. The precise nature of the surgical procedure required to deal with patients with intestinal fistulation will vary from case to case and generalisations are unlikely to be of value. There are, however, some key factors that are likely to be associated with successful reconstructive surgery. A sufficient period of time needs to be set aside for these technically-demanding procedures, which require senior surgical input and an unhurried and gentle surgical technique. Dissection should be sharp, rather than blunt, in order to avoid inadvertent bowel injury resulting in enterotomies and the possibility of further postoperative fistulation. Fistulating segments of bowel should be resected and not bypassed, to avoid blind loops, bacterial overgrowth and dysfunction of the remnant intestine. Careful anastomotic technique is vital and anastomoses should be avoided in hypoalbuminaemic $(<30 \mathrm{~g} / \mathrm{l})$ patients. Finally, anastomoses should not be undertaken or left in abscess cavities, as this practice is likely to lead to refistulation.

\section{Prognosis of acute intestinal failure}

It is neither possible nor helpful to make generalised comments concerning the prognosis of a condition that can vary from delayed recovery of intestinal function after an otherwise uncomplicated aortic vascular graft to severe abdominal sepsis in patients with multiple organ failure in the intensive care unit. The principal criteria by which the performance of surgeons managing patients with intestinal failure can be measured are hospital mortality rate and the frequency with which patients avoid chronic intestinal failure (see later). Hospital mortality rates as high as $13 \%$ have been recorded in patients with complex acute intestinal failure, even after management in a specialised national referral centre (Scott et al. 1991) and, although there are no published data, the prognosis for patients in routine surgical practice (where such patients are relatively uncommon) is likely to be considerably worse. The principal factors that determine prognosis are age, co-morbidity and the presence of severe abdominal sepsis. The continued development of specialised units for the management of patients with complicated acute intestinal failure has been associated with a steady improvement in outcome, with hospital mortality rates of $\leq 2 \%$ recorded, and $>50 \%$ of patients restored to intestinal continuity without the need for parenteral nutrition (A Bradley and JL Shaffer, personal communication).

\section{Chronic intestinal failure}

In contrast to acute intestinal failure, the role of surgery in the management of patients who have irreversible intestinal failure is far less clear. Surgical treatment is of relevance to both prevention and treatment of chronic intestinal failure.

\section{Prevention of short bowel syndrome}

In many cases (see earlier) complications of surgical treatment may actually result in such extensive resection of small intestine that chronic intestinal failure becomes inevitable. These considerations are of particular relevance for the treatment of patients with Crohn's disease, who comprise the single largest diagnostic group of patients on prolonged home parenteral nutrition in the UK. While massive small intestinal resection may be inevitable after mesenteric vascular thrombosis, timely conservative surgery, with surgical techniques that preserve intestinal length such as strictureplasty (Taschieri et al. 1997), may substantially reduce the incidence of short bowel syndrome in Crohn's disease, in which frequent disease recrudescence may otherwise result in repeated bowel resection. Perhaps of even greater importance to the prevention of short bowel syndrome is avoidance of intra-abdominal sepsis and post-operative fistulation in patients with Crohn's disease. A recent study has demonstrated that $>60 \%$ of a large series of patients with 
short bowel syndrome due to Crohn's disease developed chronic intestinal failure as a result of repeated emergency surgery to deal with post-operative intra-abdominal infection, rather than because of repeated uncomplicated resection (Agwunobi et al. 2001).

\section{Treatment of short bowel syndrome}

Surgery for treatment of chronic intestinal failure can be undertaken to restore intestinal continuity, to lengthen the intestine, delay intestinal transit or improve its function and, finally, to replace intestine (transplantation). Unlike restoration of continuity, the other surgical manoeuvres are only undertaken when it is clear that there are no prospects of additional intestinal adaptation or available medical measures that could be adopted to improve intestinal function. In addition, it should be noted that clinical experience of measures to lengthen and/or improve the function of the short small bowel has been confined largely to highly-selected groups of patients, with children featuring predominantly (Thompson, 2001).

\section{Restoration of intestinal continuity}

The most obvious surgical strategy for management of chronic intestinal failure where there is defunctioned intestine distal to a stoma is simply to restore intestinal continuity. There is evidence that this strategy not only has direct effects by recruiting additional absorptive area, but also indirect effects related to trophic effects on the proximal intestine and a 'braking' effect on intestinal motility, possibly as a result of secretion of peptides, including glucagon-like peptide 2 (Jeppesen et al. 2000). For example, little or no jejujnal adaptation is seen in the presence of an end-jejunostomy, whereas marked adaptation is observed if the jejunum is anastomosed to the colon (Goodlad et al. 2001). Jejuno-ileal and jeuno-colic anastomoses are said to be equivalent to recruitment of an additional 0.80 and $0.25 \mathrm{~m}$ small intestine respectively (Carbonnel et al. 1996). Although some reports have indicated that only a minority of patients with a short small intestine and an intact colon will undergo satisfactory restoration of continuity, this experience has by no means been universal. Anastomosing even a short length of jejunum to the colon should probably be considered wherever possible, because of the likely benefits associated with fluid and electrolyte balance and energy absorption. There are, however, potential problems associated with restoration of intestinal continuity. Many patients experience diarrhoea, which may require substantial dietary modification and addition of bile-salt-binding agents such as cholestyramine. There is also a substantial incidence of renal calculi in patients with a short bowel and colon in continuity, associated with oxaluria.

\section{Improving the function of the residual intestine}

A variety of surgical procedures have been described, all aimed at optimising the function of the residual intestine, increasing intestinal length and, in particular, slowing transit. In some patients with short bowel syndrome progressive dilatation of the residual small intestine occurs, possibly in relation to the process of adaptation. In some adults strictures may develop leading to obstruction, whereas motility abnormalities leading to 'pseudo-obstruction' have been described in children (Thompson, 2001). Dilatation of residual small bowel may be associated with bacterial overgrowth that impairs absorptive function, and strictureplasty or tapering of dilated segments has been said to be of benefit under these circumstances. Many techniques have been devised to delay intestinal transit (Thompson, 2001), in the hope that the increased contact time between the mucosa and the lumen contents may facilitate improved absorption. Reversal of segments of small intestine (Pigot et al. 1990), construction of intestinal valves (Thompson et al. 1995) and recirculating bowel loops (Watkins et al. 1984), interposition of colonic segments (Garcia et al. 1981) and implantation of reversed electrical pacing devices (Devine \& Kelly, 1989) have all been employed in order to improve the efficiency of intestinal absorption. Experience of these procedures varies considerably (Fig. 3), with reversed intestinal segments being the most extensively evaluated procedure and appearing to be the most effective (at least in the short-term). In general, these procedures are only appropriate to a small percentage of patients, notably those with a small bowel remnant of $>0.90 \mathrm{~m}$ in whom adaptation is complete and additional small or large intestine cannot be brought into play by conventional surgical reconstruction.

\section{Increasing functional intestinal length}

Bianchi (1984) devised an ingenious technique for lengthening dilated small bowel in children with short bowel syndrome. The technique is based on the observation that in children the two leaves of the small bowel mesentery are often incompletely fused, allowing the vessels passing to the two sides of the intestine to be separated and the dilated bowel reduced in diameter but lengthened. The Bianchi (1984) procedure appears to be associated with a marked improvement in nutritional status in $>90 \%$ of patients (Pokorny \& Fowler, 1991), and has been combined with valve procedures to induce initial dilatation, thus enabling later lengthening to be undertaken (Thompson, 2001). Intestinal lengthening procedures are usually reserved for children with $<0.90 \mathrm{~m}$ intestine and dilatation of small bowel to $>40 \mathrm{~mm}$, although novel techniques are being developed that may allow tissue-engineered intestine and serosal patches to be used to increase functional intestinal length.

\section{Summary}

Acute intestinal failure is a common clinical entity that usually resolves spontaneously, provided adequate nutritional and metabolic support are provided. Surgery plays a key role in the management of patients with acute intestinal failure, particularly when acute intestinal failure is associated with sepsis. The relative rarity and the immense clinical complexity of complicated acute intestinal failure may justify the continued evolution of specialised units for the management of such patients. In contrast, chronic intestinal failure is relatively uncommon. Surgery may play an important role in prevention but, beyond restoration of intestinal continuity, the role of surgical treatment for established chronic intestinal 


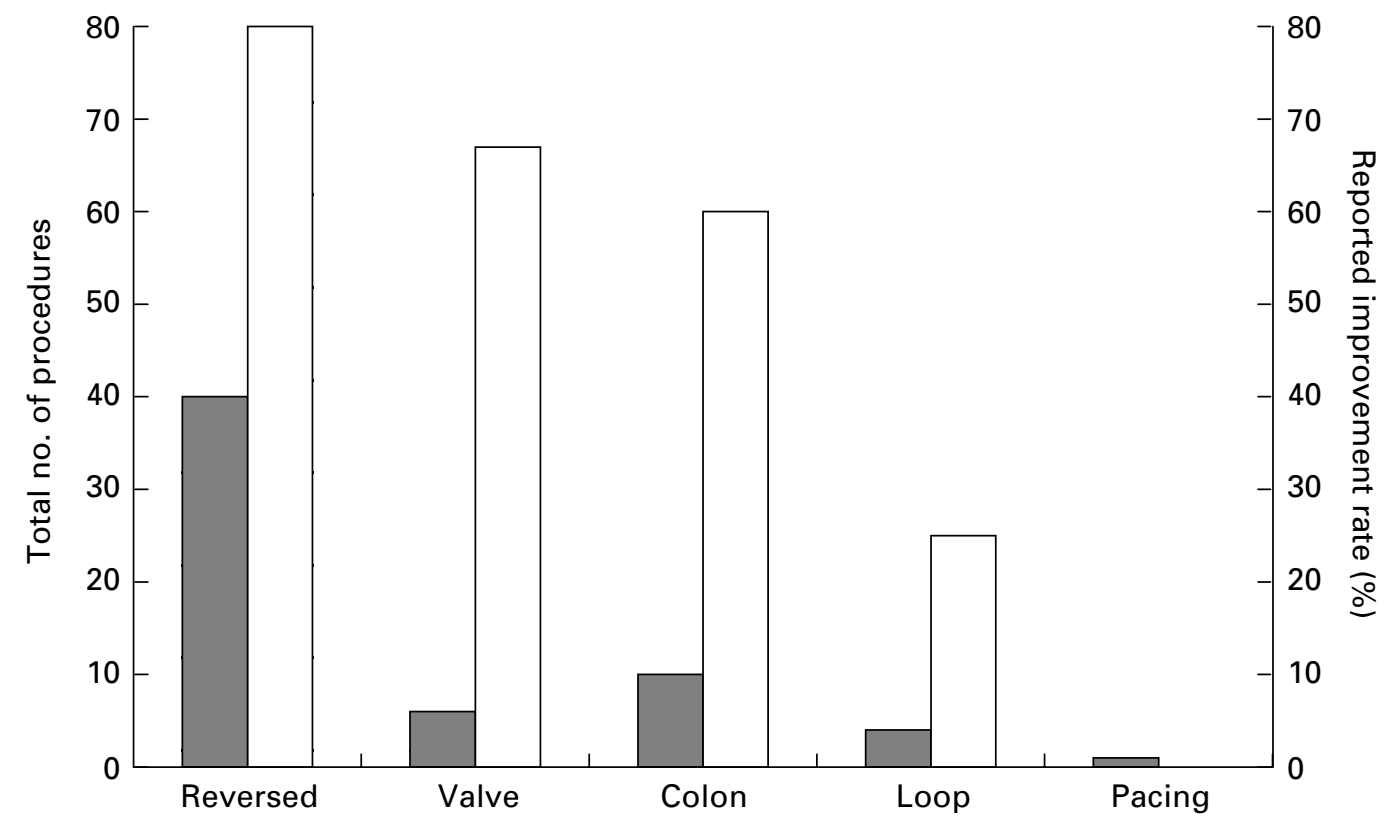

Fig. 3. Experience and results of surgery for chronic intestinal failure. ( $\square$ ), Total numbers of procedures undertaken; $\square$ ), reported improvement rates. Reversed, reversal of segments of small intestine; valve, construction of intestinal valves; colon, interposition of colonic segments; loop, construction of recirculating bowel loops; pacing, implantation of reversed electrical pacing devices. (Adapted from Thompson, 2001.)

failure continues to remain unclear and likely to evolve further in the hands of specialist units treating relatively small numbers of patients.

\section{References}

Agwunobi AO, Carlson GL, Anderson ID, Irving MH \& Scott NA (2001) Mechanisms of intestinal failure in Crohn's disease. Diseases of the Colon and Rectum 44, 1834-1837.

Bianchi A (1984) Intestinal lengthening: An experimental and clinical review. Journal of the Royal Society of Medicine 77, $35-41$.

Bone RC, Balk RA, Cerra FB, Dellinger RP, Fein AM, Knaus WA, Schein RM \& Sibbald WJ (1992) Definitions for sepsis and organ failure and guidelines for the use of innovative therapies in sepsis. Chest 101, 1644-1655.

Carbonnel F, Cosnes J \& Chevret S (1996) The role of anatomic factors in nutritional autonomy after extensive small bowel resection. Journal of Parenteral and Enteral Nutrition 20, 275-280.

Carlson GL (1998) Nutrient induced thermogenesis. In Clinical Endocrinology and Metabolism: Energy Metabolism in Trauma, pp. 603-615 [J Wernerman and RA Little, editors]. London: Balliere-Tindall.

Carlson GL (2001) Surgical causes and management. In Intestinal Failure, pp. 39-49 [JMD Nightingale, editor]. London: Greenwich Medical Media.

Carlson GL \& Irving MH (1997) Infection: recognition and management of infection in surgical patients. In Critical Care of the Surgical Patient - A Companion to Bailey and Loves' Surgery, pp. 273-290 [G Hanson, editor]. London: Chapman and Hall Medical.

Carlson GL \& Irving MH (2000) Intestinal fistulas. In Oxford Textbook of Surgery, pp. 1369-1374 [PJ Morris and WC Wood, editors]. Oxford: Oxford University Press.
Carlson GL \& Little RA (1992) The pathophysiology and pattern of the hormonal response to severe sepsis. In Endocrine Consequences of Critical Illness, pp. 57-69 [JM Boles, editor]. Paris: Arnette.

Carlson GL \& Little RA (1994) Insulin resistance and tissue fuels. In Organ Metabolism and Nutrition: Ideas for Critical Care, pp. 49-69 [JM Kinney, editor]. New York: Raven Press.

Chandran VP \& Sim AJ (1991) Nutritional support in acute intestinal failure. Bailliere's Clinical Gastroenterology 5, 841-860.

Devine RM \& Kelly KA (1989) Surgical therapy for the short bowel syndrome. Gastroenterology Clinics of North America 18, 603-617.

Fleming CR \& Remington M (1981) Intestinal failure. In Nutrition and the Surgical Patient, pp. 219-235 [GL Hill, editor]. New York: Churchill Livingstone.

Garcia VF, Templeton JM, Eichelberger MR, Koop CE \& Vinograd I (1981) Colonic interposition for the short bowel syndrome. Journal of Pediatric Surgery 16, 994-995.

Gerzof SG \& Oates ME (1988) Imaging techniques for infections in the surgical patient. Surgical Clinics of North America $\mathbf{6 8}$, $147-166$.

Goodlad R, Nightingale J \& Playford R (2001) Intestinal adaptation. In Intestinal Failure, pp. 243-260 [JMD Nightingale, editor]. London: Greenwich Medical Media.

Hau T, Ohmann C, Womershauser A, Wacha H \& Yang Q (1995) Planned relaparotomy versus relaparotomy on demand in the treatment of intraabdominal infections. The Peritonitis Study Group of the Surgical Infection Society-Europe. Archives of Surgery 130, 1193-1196.

Hughes S, Myers A \& Carlson G (2001) Care of intestinal stoma and enterocutaneous fistula. In Intestinal Failure, pp. 51-63 [JMD Nightingale, editor]. London: Greenwich Medical Media.

Jennings WC, Wood CD \& Guernsey JM (1982) Continuous postoperative peritoneal lavage in the treatment of peritoneal sepsis. Diseases of the Colon and Rectum 25, 641-643. 
Jeppesen PB, Hartmann B, Thulesen J, Hansen BS, Holst JJ, Poulsen SS \& Mortensen PB (2000) Elevated plasma glucagonlike peptide 1 and 2 concentrations in ileum-resected short bowel patients with a preserved colon. Gut 47, 370-376.

Levy EP, Frileux S, Sandrucci JM, Ollivier JP, Masini J, Cosnes L, Hannoun D \& Parc R (1988) Continuous enteral nutrition during the early adaptive stage of the short bowel syndrome. British Journal of Surgery 75, 549-553.

May JP, Murchan J, MacFie J, Sedman P, Donat R, Palmer D \& Mitchell CJ (1996) Prospective study of the aetiology of infusion phlebitis and line failure during peripheral parenteral nutrition. British Journal of Surgery 83, 1091-1094.

Mughal MM, Bancewicz J \& Irving MH (1986) Laparostomy. A technique for the management of intractable abdominal sepsis. British Journal of Surgery 73, 253-259.

Nightingale JMD (editor) (2001) Definition and classification of intestinal failure. In Intestinal Failure, pp. ix-x.London: Greenwich Medical Media.

Pettigrew RA \& Hill GL (1984) Therapeutic nutrition. In Textbook of Gastroenterology, pp. 1227-1245 [IAD Bouchier, RN Allan, HJF Hodgson and MRB Keighley, editors]. London: BailliereTindall.

Pigot FB, Messing B, Shaussade S, Pfeiffer A, Pouliguen Y \& Jiau R (1990) Severe short bowel syndrome with a surgically reversed small bowel segment. Digestive Diseases and Sciences 35, 137-144.

Plusa SM, Horsman R, Kendall-Smith S, Webster N \& Primrose JN (1998) Fine bore cannulas for peripheral intravenous nutrition: polyurethane or silicone? Annals of the Royal College of Surgeons Edinburgh 80, 154-156.

Pokorny WJ \& Fowler CL (1991) Isoperistaltic intestinal lengthening for short bowel syndrome. Surgery Gynecology and Obstetrics 173, 39-43.

Polk HC \& Fry DE (1980) Radical peritoneal debridement for established peritonitis. The results of a prospective randomized clinical trial. Annals of Surgery 192, 350-355.
Scott NA, Leinhardt DJ, O'Hanrahan T, Finnegan S, Shaffer JL \& Irving MH (1991) Spectrum of intestinal failure in a specialised unit. Lancet 337, 471-473.

Scripcariu V, Carlson G, Bancewicz J, Irving MH \& Scott NA (1994) Reconstructive abdominal operations after laparostomy and multiple repeat laparotomies for severe intra-abdominal infection. British Journal of Surgery 81, 1475-1478.

Shaw JHF \& Koea JB (1993) Metabolic basis for the management of the septic surgical patient. World Journal of Surgery 17, 154-164.

Soeters PB, Ebeid AM \& Fischer JE (1979) Review of 404 patients with gastrointestinal fistulas: Impact of parenteral nutrition. Annals of Surgery 190, 189-202.

Streat SJ, Beddoe AH \& Hill GL (1987) Aggressive nutritional support does not prevent protein loss despite fat gain in septic intensive care patients. Journal of Trauma 27, 262-266.

Taschieri AM, Elli M, Danelli PG, Molteni B, Rovati M \& Porro GB (1997) Description of new 'bowel-sparing' techniques for long strictures of Crohn's disease. American Journal of Surgery 173, 509-512.

Thompson JS (2001) Surgery for patients with a short bowel. In Intestinal Failure, pp. 515-528 [JMD Nightingale, editor]. London: Greenwich Medical Media.

Thompson JS, Langnas AN, Pinch LW, Kaufman S, Quigley EMM \& Vanderhoof JA (1995) Surgical approach to the short bowel syndrome. Experience in a population of 160 patients. Annals of Surgery 222, 600-607.

Tighe MJ, Wong C, Martin IG \& McMahon MJ (1995) Do heparin, hydrocortisone and glyceryl trinitrate influence thrombophlebitis during full intravenous nutrition via a peripheral vein? Journal of Parenteral and Enteral Nutrition 19, 507-509.

Watkins RM, Dennison AR \& Collin J (1984) Do intestinal pouches have a role in the treatment of short bowel syndrome? British Journal of Surgery 71, 384-386.

Williams N, Wales S \& Irving MH (1996) Prolonged peripheral parenteral nutrition with an ultrafine cannula and low osmolality feed. British Journal of Surgery 83, 114-116. 